\title{
On the Performance of Explorative Artificial Bee Colony Algorithm for Numeric Function Optimization
}

\author{
Tanveer Ahmed Belal \\ Ahsanullah University of Science \\ and Technology \\ Dhaka-1208, Bangladesh
}

\author{
Md. Shahriar Rahman \\ Ahsanullah University of Science \\ and Technology \\ Dhaka-1208, Bangladesh
}

\author{
Mohammad Shafiul Alam \\ Ahsanullah University of Science \\ and Technology \\ Dhaka-1208, Bangladesh
}

\begin{abstract}
The Explorative Artificial Bee Colony (EABC) algorithm is a recently introduced swarm intelligence based algorithm that has been successfully tested to optimize only a limited number of multimodal functions. This paper evaluates EABC on a larger number of benchmark functions, including both unimodal and multimodal functions. EABC is an improved variant of the Artificial Bee Colony (ABC) algorithm. A major problem with the basic $\mathrm{ABC}$ algorithm is that it is more aligned towards exploitations, rather than explorations, which often leads to premature convergence and fitness stagnation. The improved variant - EABC tries to increase the degree of explorations of $\mathrm{ABC}$ by introducing more randomness during its perturbation operations. Besides, EABC customizes the degree of exploitations and explorations at the individual solution level, separately for each candidate solution of the bee population. EABC also introduces a crossover operation that assists the explorative perturbation operation of EABC. This paper extends the experimental studies on EABC by evaluating it on as many as 13 complex, high dimensional benchmark functions, including both unimodal and multimodal, separable and non-separable functions. The results are compared with the basic $\mathrm{ABC}$ algorithm. The comparison demonstrates that $\mathrm{EABC}$ often performs better optimization than the original $\mathrm{ABC}$ algorithm, which indicates the effectiveness of its more explorative operations.
\end{abstract}

\section{Keywords}

Explorative artificial bee colony algorithm; Exploration and exploitation; Continuous function optimization

\section{INTRODUCTION}

The Artificial Bee Colony (ABC) algorithm [1] is a recently introduced swarm intelligence based algorithm inspired by the intelligent food foraging behavior of the honey bees found in nature. Since its advent [2], ABC and its variants have often successfully employed to wide and diverse range of problems, such as numeric optimization [3], discrete optimization [4], multi-objective optimization [5], industrial process control [6], structural design [7], design of digital IIR filters [8], PID controller [9], machine learning [10] and so on [11]. In comparison to other greedy and local search based algorithms, $\mathrm{ABC}$ is more resilient against premature convergence and fitness stagnation, because the population of candidate solutions can maintain some amount of diversity that is necessary to continue search space explorations avoiding the locally optimal points. However, it is still possible (e.g., [12] - [14]) that the evolving population of candidate solutions loses its diversity and explorative search capability too soon. This leads the candidate solutions to prematurely get trapped around the local optima of the search space. Aside from premature convergence, another problem that is faced by the $\mathrm{ABC}$ algorithm is fitness stagnation, where all the candidate solutions fail to improve their fitness values for indefinitely prolonged iterations, for no apparent reason and even without any premature convergence around the locally optimal points. The risk of premature convergence and fitness stagnation usually rises with reduced explorations and increased exploitations. But, increasing the explorations may lead to unacceptably slow convergence speed. So an adaptive and balanced mix of explorations and exploitations is often necessary for good results and sufficient convergence speed of the algorithm, especially for the complex, high dimensional, multimodal problems.

There exist a number of research works (e.g., [15] - [34]) that attempt to alter the explorative and/or exploitative properties of the basic $\mathrm{ABC}$ algorithm. However, most of them focus on altering the selection operation only. In the literature, not much has been reported to improve the basic, non-adaptive and fixed perturbation operator of $\mathrm{ABC}$. This is why the previously proposed algorithm — Explorative ABC [35] alters the exploitative perturbation operation of $\mathrm{ABC}$, as well as incorporates a number of basic techniques to increase the degree of explorations of the basic ABC algorithm. EABC also tries to bring a proper balance between its degree of exploitations and explorations. EABC searches with more randomness across the search space while the original $\mathrm{ABC}$ algorithm mostly searches towards and around the best candidate solutions. Besides, the number of parameters that are perturbed by EABC is gradually self-adapted, cycle (i.e., generation) by cycle, separately for each candidate solution, while $\mathrm{ABC}$ always uses a fixed, small (hence, exploitative) perturbation rate. The objective of EABC is to introduce more randomness during perturbations for more search space explorations, and to customize the degree of explorations and exploitations at the individual candidate solution level, by adapting the perturbation rate separately for each candidate solution of the bee population.

The rest of this paper is organized as follows. Section 2 describes the ABC algorithm in details. Section 3 presents a few improved $\mathrm{ABC}$-variants and explains how $\mathrm{EABC}$ is significantly different from them. Section 4 describes EABC in details. Section 5 provides details of the benchmark problems and compares the results of $\mathrm{ABC}$ and $\mathrm{EABC}$. Finally, section 6 concludes the paper by leaving a few suggestions for further research with EABC.

\section{THE ORIGINAL ARTIFICIAL BEE COLONY (ABC) ALGORITHM}

Honey bees in a colony show remarkable self-organization 
and co-ordination skills in their food foraging behavior. Bees have to forage over a vast area in search of good sources of food. After an initial exploration stage, more bees are employed to collect honey from the more profitable food sources whereas fewer bees are assigned to the less worthy food sources. Some scout bees are also assigned for exploration to find newer food sources. If the quality of a food source declines after some exploitation, this information is also shared with other bees so that fewer bees are now attracted to this source. After the quality of a food source falls below some threshold, the bees assigned to it abandon it. The foraging process is initiated by scout bees that start searching for flower patches suitable as food sources. Quality is usually measured as a combination of some values, such as quantity and density of sugar, ease of access, distance from the colony etc. After they return to the hive, those scout bees that found a patch with quality above some threshold, deposit their nectar and then go to the 'dance floor' to perform a dance known as the 'waggle dance'. This dance plays the key role to communicate information among the bees about the food sources. The waggle dance contains three pieces of information: i) the quality of the flower patch of this dancing bee, ii) the distance of the flower patch from the hive, iii) the direction from the hive that you have to travel in order to reach the flower. The 'onlooker' bees, waiting around the dance floor, observe the waggle dances of these 'employed' bees that have found good food sources and pick any one of them to become its 'follower' and collect nectar from its flower patch. The better a flower patch as a food source, the bigger is the number of follower bees along with its employed bee. However, if the patch is no longer good enough, it will not be advertised in the next waggle dance and the bees recruited for it as employed or follower bees will choose either to follow some other employed bee or start working as a scout bee to randomly explore the search space for finding new food source.

The ABC algorithm mimics the food foraging behavior of the honey bees with these three groups of bees: employed bees, onlookers and scouts. A bee working to forage a food source (i.e. solution) previously visited by itself and searching only around its vicinity is called an employed bee. Employed bees perform waggle dance to propagate information of its food source to other bees. A bee waiting around the dance floor to choose any of the employed bees to follow is called an onlooker. A bee randomly searching a search space for finding a food source is called a scout. For every food source, there is only one employed bee and a number of follower bees. The scout bee, after finding a good food source also becomes an employed bee. In the basic $A B C$ algorithm implementation, half of the colony is employed bees and the other half is the onlookers. Number of food sources (i.e., solutions) is equal to the number of employed bees. An employed bee whose food source is exhausted (i.e. solution has not improved after several attempts) becomes a scout. The detailed pseudocode is given below.

Step 1) Generate an initial population of $N$ individuals. Each individual is a food source (i.e. solution) and has $D$ attributes, where $D$ is the dimensionality of the problem.

Step 2) Evaluate the fitness of each individual.

Step 3) Each employed bee searches in the neighborhood of its current position to find a better food source. For each employed bee, generate a new solution, $\boldsymbol{v}_{i}$ around its current position, $\boldsymbol{x}_{\boldsymbol{i}}$ using (1).

$$
v_{i j}=x_{i j}+\varphi_{i j}\left(x_{i j}-x_{k j}\right)
$$

Here, $k \in\left\{1,2, \ldots, N_{e m p}\right\}$ and $j \in\{1,2, \ldots, D\}$ are randomly chosen indices. $N_{e m p}$ is the number of employed bees. $\Phi_{i j}$ is a uniform random number generated from the range $[-1,1]$.

Step 4) Compute the fitness of both $x_{i}$ and $v_{i}$. Apply greedy selection scheme to choose the better one and discard the other.

Step 5) Calculate the selection probability, $P_{i}$ for each solution, $\boldsymbol{x}_{\boldsymbol{i}}$ and normalize the probability value by (2).

$$
P_{i}=f i t_{i} / \sum_{k=1}^{N} f i t_{k}
$$

Step 6) Assign each onlooker bee to a solution, $x_{i}$ at random with probability proportional to $P_{\mathrm{i}}$

Step 7) Produce new food positions (i.e. solutions), $v_{i}$ for each onlooker bee using the employed bee $\boldsymbol{x}_{i}$ by using (1).

Step 8) Evaluate the fitness of each employed bee, $\boldsymbol{x}_{\boldsymbol{i}}$ and its produced onlooker bee, $\boldsymbol{v}_{\boldsymbol{i}}$. Apply greedy selection scheme to keep the one with better fitness and discard the other.

Step 9) If a particular solution has not been improved over a number (say, 100) of cycles, then select it for abandonment. Replace it by placing a scout bee at a food source placed uniformly at random over the entire search space using (3), i.e., for $j=1,2, \ldots, D$

$$
x_{i j}=\min _{j}+\operatorname{rand}(0,1) *\left(\max _{j}-\min _{j}\right)
$$

Step 10) Keep track of the best solution found so far.

Step 11) Check for termination. If the best solution found is acceptable or maximum number of iterations has elapsed, stop and return the best solution found so far. Otherwise go back to step 2 and repeat.

\section{EXISTING VARIANTS OF THE ABC ALGORITHM}

There exist several recent works (e.g., [16] - [34]) that try to tweak the explorative and/or exploitative properties of the basic $\mathrm{ABC}$ algorithm. For example, the cooperative $\mathrm{ABC}$ (CABC) algorithm [16] decomposes the search space into a number of subspaces and enforces more explorations by employing different bee colonies to explore the different subspaces. Another explorative variant - $\mathrm{ABC}$ with diversity strategy (DABC) [17] tries to preserve sufficient amount of diversity among the candidate solutions by switching between two different perturbation schemes. The Chaotic ABC $(\mathrm{CHABC})[18]$ is another explorative $\mathrm{ABC}$-variant that uses dynamic chaotic sequence generators, instead of random number generators, to improve the explorative characteristics of the basic $\mathrm{ABC}$ algorithm. The explorative search capacity of $\mathrm{ABC}$ may also be improved by intelligent organization of the locally optimal points [19] and using the information of the global best solution, as in the Gbest-guided ABC (GABC) [20] algorithm. The Hooke Jeeves ABC (HJABC) [21] is another improved $\mathrm{ABC}$-variant that hybridizes the Hooke Jeeves pattern search technique with the basic $A B C$ algorithm. The elitist $\mathrm{ABC}$ (EABC) [22] is a hybrid $\mathrm{ABC}$ variant which hybridizes $\mathrm{ABC}$ with two different local search operators to intensify the exploitations around the best solutions. Quan and Shi [23] reported improvement of the convergence speed by introducing an exploitative search 
iteration operator based on the fixed point theorem of contractive mapping. Qingxian and Haijun [24] employed the Boltzmann selection scheme and introduced an improved initialization scheme to improve the convergence speed. The hybrid crossover based $\mathrm{ABC}$ (CBABC) [25] is an exploitative variant that strengthens the exploitation phase of $\mathrm{ABC}$ by using a crossover operation. Another recent ABC-variant NABC [26] alters the search pattern of employed and onlooker bees by searching around neighborhood of best solutions. JA-ABC [27] tries to improve average fitness of bee population by replacing poor solutions with perturbations of the fittest solution, which makes it exploitative. Some other recently introduced $\mathrm{ABC}$ variants can be found in the literature (e.g., [28] - [34]), but almost all of them come with some limitations, such as inadequate degree of explorations [28]-[30], poor exploitations [31], slower rate of convergence of the algorithm [32] and increased computational complexity [33], [34].

However, most of these $\mathrm{ABC}$-variants try to improve either the explorative or the exploitative properties if the basic $\mathrm{ABC}$ algorithm, while very few of them (e.g., [17]) tries to achieve a proper balance between explorations and exploitations. The explorative enhancements are usually based on more explorative perturbation, selection and/or initialization (e.g., [18], [19]) or employing some technique to maintain more population diversity (e.g., [16], [17]), while the exploitative developments are usually based on increasing the local search operations around the best candidate solutions ([21]-[22], [26]-[27]). Another limitation of all these ABC-variants (e.g., [16]-[34]) is that they do not consider the individual explorative/exploitative needs of the candidate solutions; rather they treat all the candidate solutions equally, employing some population-wide uniform strategy, identically on all candidate solutions. EABC differs from all these algorithms in both these aspects. First, EABC improves both explorations and exploitations by introducing more randomness during perturbations and by incorporating a crossover operation after perturbations. Second, EABC customizes explorations and exploitations separately for every candidate solution $\boldsymbol{x}_{\boldsymbol{i}}$ of the bee population by introducing and separately maintaining a control parameter $R_{i}$ for each $\boldsymbol{x}_{i}$. Third, EABC tries to bring a proper balance between explorations and exploitations by abandoning the exploitative onlooker bee phase and the explorative scout bee phase of the original ABC algorithm. All these modifications are explained in details in the following section.

\section{THE EXPLORATIVE ABC (EABC) ALGORITHM}

Although EABC is previously introduced and explained in details in [35], we are repeating here the same section (4) of [35] for the sake of clarity and self-containment of this paper. There exist several aspects on which EABC is different from the original ABC algorithm. Firstly, EABC uses the following perturbation operation (4), which is different from the perturbation eq. (1) of the original $\mathrm{ABC}$ algorithm. For each employed bee $\boldsymbol{x}_{i, G}$ of the current generation $G$, EABC first produces a perturbed vector $\boldsymbol{w}_{i, G+1}$ using (4).

$$
\boldsymbol{w}_{i, G+1}=\boldsymbol{x}_{r 1, G}+\varphi_{i, G+1}\left(\boldsymbol{x}_{r 2, G}-\boldsymbol{x}_{r 3, G}\right)
$$

Here, $r_{1}, r_{2}, r_{3}$ are randomly chosen indices from $[1, N]$ that are mutually different and also different from the current index $i$. To be more precise, $r_{1} \neq r_{2} \neq r_{3} \neq i$. The $\varphi_{i, G+1}$ is a uniform, random variable that picks its values randomly from
$[0,2]$. The details of how the values of $\varphi_{i, G+1}$ are produced randomly are explained in a subsequent paragraph. If we compare the perturbation eq. (4) of EABC with the previously used eq. (1) by $A B C$, we can observe that $E A B C$ employs relatively higher degree of randomness in (4) by using three random indices $r_{1}, r_{2}, r_{3}$ in order to ensure higher degree of search space explorations.

Secondly, EABC introduces a crossover operation to better combine the information of the original candidate solution $\boldsymbol{x}_{i, G}$ and perturbed candidate solution $\boldsymbol{w}_{i, G+1}$ to produce the new candidate solution $\boldsymbol{v}_{i, G+1}$. Each parameter of $\boldsymbol{v}_{i, G+1}$ is selected at random, either from $x_{i, G}$ (with probability $=R_{i, G+1}$ ) or from $\boldsymbol{w}_{i, G+1}$ (with probability $=1-R_{i, G+1}$ ). However, EABC also ensures that at least one parameter is selected from the perturbed vector $\boldsymbol{w}_{i, G+1}$; otherwise the new candidate solution $\boldsymbol{v}_{i, G+1}$ would be identical to its parent candidate solution $\boldsymbol{x}_{i, G}$. The details of how the value of $R_{i, G+1}$ is produced and maintained are explained in a subsequent paragraph. After producing $\boldsymbol{v}_{i, G+1}$, EABC employs a greedy selection between the original candidate solution $\boldsymbol{x}_{i, G}$ and new candidate solution $v_{i, G+1}$, similar to the step (4) of the original ABC algorithm.

Thirdly, to customize the degree of explorations and exploitations at the individual solution level, EABC maintains the scaling factor $\varphi_{i}$ and crossover rate $R_{i}$, separately for every candidate solution $\boldsymbol{x}_{i}$. The values of $\varphi_{i}$ and $R_{i}$ are gradually adapted, generation by generation, separately for each candidate solution, by using the following Eqs. (5) and (6).

$$
\begin{aligned}
& \varphi_{i, G+1}= \begin{cases}\varphi_{\text {min }}+\operatorname{rand}(0,1) *\left(\varphi_{\text {max }}-\varphi_{\text {min }}\right) ; & \text { if } \operatorname{rand}(0,1) \leq p_{1} \\
\varphi_{i, G} & \text { otherwise }\end{cases} \\
& R_{i, G+1}= \begin{cases}R_{\text {min }}+\operatorname{rand}(0,1) *\left(R_{\text {max }}-R_{\text {min }}\right) ; & \text { if } \operatorname{rand}(0,1) \leq p_{2} \\
R_{i, G} & \text { otherwise }\end{cases}
\end{aligned}
$$

Both the above equations put proper emphasis not only on search space explorations by innovation (i.e., trying new values for $\varphi_{i}$ and $R_{i}$, with probability $p_{1}$ and $p_{2}$, respectively), but also on search space exploitations by inheritance (i.e., using the same value of parent $\boldsymbol{x}_{i}$, with probability $=1-p_{1}$ and $1-p_{2}$ for $\varphi_{i}$ and $R_{i}$, respectively). Together they try to balance between the degree of explorations and exploitations, by using a suitable value of all these parameters $-p_{1}$ and $p_{2}, \varphi_{\min }$ and $\varphi_{\max }, R_{\min }$ and $R_{\max }$. EABC uses the following parameter values: $\quad p_{1}=p_{2}=0.1, \varphi_{\min }=0.1, \quad \varphi_{\max }=0.9, \quad R_{\min }=0 \quad$ and $R_{\max }=1.0$. All these values are picked after some initial experiments (by trial and error) and are not meant to be the optimum.

Fourthly, with all the above explorative and exploitative measures, we have found (with some trial and error) that the necessity of using separate onlooker bee phase and scout bee phase (as used by the original $\mathrm{ABC}$ algorithm) does not exist anymore for the proposed EABC algorithm. So, EABC has abandoned both the onlooker bee phase (i.e., steps 5-8) and scout bee phase (i.e., step 9) of the original ABC algorithm.

\section{EXPERIMENTAL STUDIES}

To evaluate the performance of the proposed EABC algorithm, we have used a standard benchmark suite on continuous function optimization problems, consisting of 13 high dimensional functions [1], [2], [18]. Table 1 presents a 
brief overview on each of the 13 standard benchmark functions. More details on each benchmark function can be found in [1]. The benchmark suite consists of both unimodal $\left(f_{1}-f_{7}\right)$ and multimodal $\left(f_{8}-f_{13}\right)$, separable (e.g., $\left.f_{1}, f_{4}\right)$ and non-separable (e.g., $f_{2}, f_{3}$ ) functions. EABC is already tested on the six multimodal functions in its previous study [35]. However, some of the unimodal functions can also pose great complexity and challenge (e.g., Rosenbrock function $f_{5}$ ) to any optimization algorithm. In case of a multimodal function, the search algorithm must possess both exploitative and explorative characteristics so that it can explore the locally optimal points without being trapped around any of them. Some of the multimodal functions can have hundreds of local minima, even when the dimensionality is just two or three. The number of local optima usually increases exponentially with the number of dimensions, which makes their optimization extremely difficult. For example, the Ackley function $f_{10}$ has one narrow global minimum basin, but with exponentially many minor local minima. The Griewank function $f_{11}$ has a component creating linkage among the variables, which complicates the search by perturbing any subset of the variables. Any technique that tries to optimize each variable separately without considering the others will fail with this function. The difficulty for the Schwefel function $f_{8}$ arises from its deep local minima which are far from the single global minimum. All the multimodal functions $f_{8}-f_{13}$ have exponentially many local minima and the number of local minima increases exponentially with the high dimensionality (i.e., $D=30$ ), making them extremely difficult for any algorithm to be explored and optimized without being trapped around the locally optimal points of the search space.

Table 1: The 13 continuous benchmark functions used in our experimental studies. Here, $D$ : dimensionality of the function, $S$ : search space, $f_{\text {min }}$ : function value at the global minimum, $C$ : function characteristics with the values - $U$ : Unimodal, $M$ : Multimodal, $S$ : Separable, $N$ : Non-separable.

\begin{tabular}{|c|c|c|c|c|c|}
\hline No & Function & C & $D$ & $S$ & $f_{\min }$ \\
\hline$f_{1}$ & Sphere & $U S$ & 30 & {$[-100,100]^{D}$} & 0 \\
\hline$f_{2}$ & Schwefel 2.22 & $U N$ & 30 & {$[-10,10]^{D}$} & 0 \\
\hline$f_{3}$ & Schwefel 1.2 & $U N$ & 30 & {$[-100,100]^{D}$} & 0 \\
\hline$f_{4}$ & Schwefel 2.21 & $U S$ & 30 & {$[-10,10]^{D}$} & 0 \\
\hline$f_{5}$ & Rosenbrock & $U N$ & 30 & {$[-30,30]^{D}$} & 0 \\
\hline$f_{6}$ & Step & $U S$ & 30 & {$[-100,100]^{D}$} & 0 \\
\hline$f_{7}$ & Quartic & $U S$ & 30 & {$[-1.28,1.28]^{D}$} & 0 \\
\hline$f_{8}$ & Schwefel 2.26 & $M S$ & 30 & {$[-500,500]^{D}$} & -12569.5 \\
\hline$f_{9}$ & Rastrigin & $M S$ & 30 & {$[-5.12,5.12]^{D}$} & 0 \\
\hline$f_{10}$ & Ackley & $M N$ & 30 & {$[-32,32]^{D}$} & 0 \\
\hline$f_{11}$ & Griewank & $M N$ & 30 & {$[-600,600]^{D}$} & 0 \\
\hline$f_{12}$ & Penalized & $M N$ & 30 & {$[-50,50]^{D}$} & 0 \\
\hline$f_{13}$ & Penalized2 & $M N$ & 30 & {$[-50,50]^{D}$} & 0 \\
\hline
\end{tabular}


Table 2: Performance of the proposed algorithm EABC, compared to the basic ABC algorithm on the standard benchmark functions. Results are averaged over 50 independent runs. Better performance on each function is marked with boldface font. In case the performance difference is not significant by $t$-Test with at least $99 \%$ level of confidence (i.e., $\alpha=0.99)$, it is marked by "Similar" at the rightmost column

\begin{tabular}{|c|c|c|c|c|c|c|}
\hline \multirow{2}{*}{ No } & \multirow{2}{*}{$f_{\min }$} & \multicolumn{2}{|c|}{$\mathbf{A B C}$} & \multicolumn{2}{|c|}{ ЕABC } & \multirow{2}{*}{$\begin{array}{l}\text { Better Performance } \\
(t \text {-Test with } \alpha=0.99)\end{array}$} \\
\hline & & Mean Error & Std. Dev. & Mean Error & Std. Dev. & \\
\hline$f_{1}$ & 0 & $7.58 \mathrm{e}-16$ & $2.61 \mathrm{e}-17$ & $4.01 \mathrm{e}-29$ & $7.25 \mathrm{e}-30$ & EABC \\
\hline$f_{2}$ & 0 & $6.06 \mathrm{e}-12$ & $1.59 \mathrm{e}-12$ & $4.30 \mathrm{e}-17$ & $8.89 \mathrm{e}-18$ & EABC \\
\hline$f_{3}$ & 0 & $2.97 \mathrm{e}+01$ & $3.94 \mathrm{e}+00$ & $8.32 \mathrm{e}-02$ & $1.52 \mathrm{e}-02$ & EABC \\
\hline$f_{4}$ & 0 & $6.57 \mathrm{e}+03$ & $5.99 \mathrm{e}+02$ & $4.93 \mathrm{e}-04$ & $6.85 \mathrm{e}-05$ & EABC \\
\hline$f_{5}$ & 0 & $1.48 \mathrm{e}-01$ & $1.28 \mathrm{e}-01$ & $1.95 \mathrm{e}+01$ & $3.72 \mathrm{e}+00$ & $\mathrm{ABC}$ \\
\hline$f_{6}$ & 0 & 0 & 0 & 0 & 0 & Similar \\
\hline$f_{7}$ & 0 & $2.08 \mathrm{e}-01$ & $3.26 \mathrm{e}-02$ & $5.69 \mathrm{e}-03$ & $6.12 \mathrm{e}-04$ & EABC \\
\hline$f_{8}$ & -12569.5 & $1.86 \mathrm{e}+01$ & $5.21 \mathrm{e}+00$ & $1.034-02$ & $3.66-03$ & EABC \\
\hline$f_{9}$ & 0 & $7.15 \mathrm{e}-16$ & $6.44 \mathrm{e}-17$ & $2.33 \mathrm{e}-31$ & $3.58 \mathrm{e}-32$ & EABC \\
\hline$f_{10}$ & 0 & $8.97 \mathrm{e}-12$ & $1.15 \mathrm{e}-12$ & $1.12 \mathrm{e}-14$ & $4.05 \mathrm{e}-15$ & EABC \\
\hline$f_{11}$ & 0 & $6.39 \mathrm{e}-16$ & $9.25 \mathrm{e}-17$ & $1.09 \mathrm{e}-30$ & $2.99 \mathrm{e}-31$ & EABC \\
\hline$f_{12}$ & 0 & $7.03 e-16$ & $1.86 \mathrm{e}-16$ & $7.39 \mathrm{e}-14$ & $1.76 \mathrm{e}-14$ & $\mathrm{ABC}$ \\
\hline$f_{13}$ & 0 & $2.61 \mathrm{e}-03$ & $8.36 \mathrm{e}-17$ & $2.61 \mathrm{e}-03$ & $8.22 \mathrm{e}-17$ & Similar \\
\hline
\end{tabular}

Table 2 presents the results of $\mathrm{EABC}$ with the basic $\mathrm{ABC}$ [1] algorithm. For both the unimodal (i.e., $f_{1}-f_{7}$ ) and multimodal functions (i.e., $f_{8}-f_{13}$ ), the common parameters are set as population size $N=100$, maximum number of generations $M G N=1500$ and limit $=100$. The other parameters of EABC are set as: $p_{1}=p_{2}=0.1, \varphi_{\min }=0.1, \varphi_{\max }=0.9, R_{\min }=0$ and $R_{\max }=1.0$. The initial values of $\varphi_{i}$ and $R_{i}$ are set to 0.5 and 0.9 , respectively for all the candidate solutions $\boldsymbol{x}_{i}$ of the initial bee population. Each algorithm has made 50 independent runs on each function. The mean and standard deviation of the error values (i.e., the difference between best found solution and global minimum) found from the different runs are reported in Table 2. In the following few points, we summarize our observations on the results.

- Out of the 13 functions $f_{1}-f_{13}$, EABC performs better than $\mathrm{ABC}$ on as many as nine functions, perform equally well on two, while $\mathrm{ABC}$ performs better only on the remaining two functions. Thus the overall performance of EABC is better than the basic ABC algorithm.

- For all these functions, EABC reaches very close to the global minimum value (i.e., mean error $\approx 0$, except only one function $-f_{5}$ ), while the basic $\mathrm{ABC}$ algorithm fails to reach sufficiently close to the global minimum for as many as four functions $\left(f_{3}\right.$, $f_{4}, f_{5}$ and $f_{8}$ ).
- The performance of EABC is very consistent, i.e., EABC regularly reaches very close to the global minimum, which is demonstrated by the very low standard deviation values of the errors of EABC.

- In summary, EABC is more effective than the original $\mathrm{ABC}$ algorithm on almost all of these complex, high dimensional continuous benchmark functions.

\section{CONCLUSION}

This paper extends the experimental studies on EABC by evaluating it on a number of unimodal and multimodal functions, while EABC was previously tested only on a few multimodal functions [35]. EABC is an explorative variant of the basic $\mathrm{ABC}$ algorithm that incorporates more randomness and introduces self-adaptive strategies on the basic perturbation operation of the original $\mathrm{ABC}$ algorithm. The experimental results on 13 complex, high dimensional, unimodal and multimodal functions indicate that EABC can perform better than $\mathrm{ABC}$ on most of these functions.

There might be several possible ways to further improve the EABC algorithm. Firstly, EABC uses a simple strategy to adapt the scaling factor and crossover rate for each candidate solution. Some more sophisticated scheme, such as some scheme parameterized by the current maturity of the search process, e.g., the current population diversity, may improve the algorithm further. Secondly, EABC puts more emphasis to increase the degree of explorations, rather than exploitations. 
Putting somewhat more emphasis on exploitations, especially around the best-so-far candidate solutions, may further improve the results. Thirdly, the quality of the final solution might be improved further by using an efficient local searcher after the execution of EABC is over. Finally, EABC has been applied only on the benchmark continuous optimization problems. It would be interesting to study how well EABC can perform on many other existing problems, especially the discrete and real world ones.

\section{ACKNOWLEDGEMENT}

This paper presents an incremental research work based on two previous works (i.e., [35], [36]). This paper reuses the text of these earlier works with the consent and guidance of their authors, for which the authors of this paper express their heartiest thanks and gratitude to all the authors of the earlier works [35], [36].

\section{REFERENCES}

[1] D. Karaboga and B. Basturk, On the performance of artificial bee colony (ABC) algorithm, Applied Soft Computing 8 (1) (2008) 687-697.

[2] D. Karaboga, An idea based on honey bee swarm for numerical optimization, Erciyes University, Kayseri, Turkey, Technical Report-TR06, 2005.

[3] D. Karaboga and B. Akay, A comparative study of artificial bee colony algorithm, Applied Mathematics and Computation 214 (1) (2009) 108-132.

[4] S. Sobti and P. Singla, Solving travelling salesman problem using bee colony based approach, International Journal of Engineering Research and Technology 2 (6) (2013) 186-189.

[5] K. Naidu, H. Mokhlis and A.H.A. Bakar, Multiobjective optimization using weighted sum Artificial Bee Colony algorithm for Load Frequency Control, International Journal of Electrical Power and Energy Systems 55 (2) (2014) 657-667.

[6] R. Mukherjee, D. Goswami and S. Chakraborty, Parametric optimization of Nd:YAG laser beam machining process using artificial bee colony algorithm, Journal of Industrial Engineering, vol. 2013, Article ID 570250, 15 pages, 2013. DOI: 10.1155/2013/570250.

[7] H. Garg, Solving structural engineering design optimization problems using an artificial bee colony algorithm, Journal of Industrial and Management Optimization, 10 (3) (2014) 777-794.

[8] Z. Zhao, D. Yin and Y. Jiang, Improved bee colony algorithm based on knowledge strategy for digital filter design, International Journal of Computer Applications, 47 (2) (2013) 241-248.

[9] A. Mishra, A. Khanna, N. Singh and V. Mishra, Speed control of DC motor using bee colony optimization, Universal Journal of Electrical and Electronic Engineering 1 (3) (2013) 68-75.

[10] A. Karegowda and M. Darshan, Optimizing feed forward neural network connection weights using artificial bee colony algorithm, International Journal of Advanced Research in Computer Science and Software Engineering 3 (7) (2013) 452-454.

[11] A. Bolaji, A. Khader, M. Betar and M. Awadallah, Bee colony algorithm, its variants and applications: A survey, Journal of Theoretical and Applied Technology 47 (2) (2013) 434-459.

[12] T. Park and K. R. Ryu, A Dual population genetic algorithm for adaptive diversity control, IEEE Trans. Evolutionary Computation 14 (6) (2010) 865-884.

[13] R. K. Ursem, Diversity guided evolutionary algorithms, in Proc. 7th Int. Conf. Parallel Problem Solving from Nature (PPSN), 2002, pp. 462-474.

[14] J. Lampinen and I. Zelinka, On stagnation of the differential evolution algorithm, in Proc. 6th Int. Mendel Conf. Soft Computing, Brno, Czech Republic, 2000, pp. 76-83.

[15] V. Tereshko, A. Loengarov, "Collective DecisionMaking in Honey Bee Foraging Dynamics", Comput. Inf. Sys. J., vol. 9, no. 3, pp. 1-7, 2005.

[16] M. Abd, A cooperative approach to the artificial bee colony algorithm, in Proc. IEEE Congress on Evolutionary Computation (CEC), 2010, pp. 1-5.

[17] W. Lee and W. Cai, A novel artificial bee colony algorithm with diversity strategy, in Proc. 7th Int. Conf. Natural Computation, 2011, pp. 1441-1444.

[18] B. Wu and S. Fan, Improved artificial bee colony algorithm with chaos, in Computer Science for Environmental Engineering and Eco-Informatics, Part I Communications in Computer and Information Science, eds. Y. Yu, Z. Yu and J. Zhao, vol. 158, 2011, pp. 51-56.

[19] L. Fenglei, D. Haijun and F. Xing, The parameter improvement of bee colony algorithm in TSP problem, Science Paper Online, Nov. 2007.

[20] G. Zhu and S. Kwong, Gbest-guided artificial bee colony algorithm for numerical function optimization, Applied Mathematics and Computation 217 (7) (2010) 31663173 .

[21] F. Kang, J. Li, Z. Ma and H. Li, Artificial bee colony algorithm with local search for numerical optimization, Journal of Software 6 (3) (2011) 490-497.

[22] E. Montes and R. Koeppel, Elitist artificial bee colony for constrained real-parameter optimization, in Proc IEEE Congress on Evolutionary Computation, 2010, pp. $1-8$.

[23] H. Quan and X. Shi, On the analysis of performance of the improved artificial bee colony algorithm, in Proc. 4th Int. Conf. Natural Computation (ICNC), 2008, pp. 654658.

[24] F. Qingxian and D. Haijun, Bee colony algorithm for the function optimization, Science Paper Online, Aug. 2008.

[25] S. Kumar, V. Sharma and R. Kumari, A novel crossover based artificial bee colony algorithm for optimization, International Journal of Computer Applications 82 (8) (2013) 18-25.

[26] Y. Xu, P. Fan and L. Yuan, A simple and efficient artificial bee colony algorithm, Mathematical Problems in Engineering, vol. 2013, Article ID 526315, 9 pages 2013. DOI: $10.1155 / 2013 / 526315$.

[27] N. Sulaiman, J. Saleh and A. Abro, A modified artificial 
bee colony (JA-ABC) optimization algorithm, in Proc. International Conference on Applied Mathematics and Computational Methods in Engineering (AMCME), 2013, pp. 74-79.

[28] A. Abro and J. Saleh, Enhanced global-best artificial bee colony optimization algorithm, in Proc. $6^{\text {th }}$ European Symposium on Computer Modeling and Simulation, 2012, pp. 95-100.

[29] W. Gao, S. Liu and L. Huang, A global best bee colony algorithm for global optimization, Journal of Computational and Applied Mathematics 236 (11) (2012) pp. 2741-2753.

[30] W. Gao and S. Liu, A modified artificial bee colony algorithm, Computers and Operations Research 39 (3) (2012) pp. 687-697.

[31] W. Gao and S. Liu, Improved artificial bee colony algorithm for global optimization, Information Processing Letters 111 (17) (2011) pp. 871-882.
[32] G. Zhu, S. Kwong, Gbest-guided bee colony algorithm for numerical optimization, Applied Mathematics and Computation 217 (7) (2010) pp. 3166-3173.

[33] A. Abro and J. Saleh, An enhanced artificial bee colony optimization algorithm, Recent Advances in Systems Science and Mathematical Modelling, ed. D.S. Nikos Mastorakis, Valeriu Prepelita, 2012: WSEAS Press.

[34] A. Banharnsakun, T. Achalakul and B. Sirinaovakul, The best-so-far selection in artificial bee colony algorithm, Applied Soft Computing 11 (2) (2011) pp. 2888-2901.

[35] S. S. Shapla, H. M. Haque and M. S. Alam, Explorative Artificial Bee Colony Algorithm: A Novel Swarm Intelligence Based Algorithm For Continuous Function Optimization, Accepted for publication in International Journal of Science and Research (IJSR) 4 (7) (2015).

[36] S. S. Hasan and F. Ahmed, Balaning Explorations with Exploitations in the Artificial Bee Colony Algorithm for Numerical Function Optimization, International Journal of Applied Information Systems 9 (1) (2015) pp. 42-48. 